\title{
Does abnormal sleep impair memory consolidation in schizophrenia?
}

\author{
Dara S. Manoach ${ }^{1,2,3 *}$ and Robert Stickgold ${ }^{3,4}$ \\ 1 Department of Psychiatry, Massachusetts General Hospital, Charlestown, MA, USA \\ 2 Athinoula A. Martinos Center for Biomedical Imaging, Charlestown, MA, USA \\ 3 Harvard Medical School, Boston, MA, USA \\ ${ }^{4}$ Department of Psychiatry, Beth Israel Deaconess Medical Center, Boston, MA, USA
}

\section{Edited by:}

Kenneth Hugdahl,

University of Bergen, Norway

\section{Reviewed by:}

Janne Grønli,

University of Bergen, Norway Kenneth Hugdahl, University of

Bergen, Norway

*Correspondence:

Dara S. Manoach, Psychiatric

Neuroimaging, Massachusetts General

Hospital, Charlestown Navy Yard, 149

13th Street, Room 2608, Charlestown,

MA 02129, USA.

e-mail:dara@nmr.mgh.harvard.edu
Although disturbed sleep is a prominent feature of schizophrenia, its relation to the pathophysiology, signs, and symptoms of schizophrenia remains poorly understood. Sleep disturbances are well known to impair cognition in healthy individuals. Yet, in spite of its ubiquity in schizophrenia, abnormal sleep has generally been overlooked as a potential contributor to cognitive deficits. Amelioration of cognitive deficits is a current priority of the schizophrenia research community, but most efforts to define, characterize, and quantify cognitive deficits focus on cross-sectional measures. While this approach provides a valid snapshot of function, there is now overwhelming evidence that critical aspects of learning and memory consolidation happen offline, both over time and with sleep. Initial memory encoding is followed by a prolonged period of consolidation, integration, and reorganization, that continues over days or even years. Much of this evolution of memories is mediated by sleep. This article briefly reviews (i) what is known about abnormal sleep in schizophrenia, (ii) sleep-dependent memory consolidation in healthy individuals, (iii) recent findings of impaired sleep-dependent memory consolidation in schizophrenia, and (iv) implications of impaired sleep-dependent memory consolidation in schizophrenia. This literature suggests that abnormal sleep in schizophrenia disrupts attention and impairs sleep-dependent memory consolidation and task automation. We conclude that these sleep-dependent impairments may contribute substantially to generalized cognitive deficits in schizophrenia. Understanding this contribution may open new avenues to ameliorating cognitive dysfunction and thereby improve outcome in schizophrenia.

Keywords: sleep, schizophrenia, procedural learning, motor skill, memory consolidation, cognition, slow wave sleep, sleep spindles

\section{INTRODUCTION}

Sleep disorders are a prominent feature of many neuropsychiatric disorders. While often viewed as secondary, as these disorders may themselves diminish sleep quality (Benca, 1996), sleep deprivation can precipitate psychosis (Tyler, 1955; Wright, 1993, but see, Kahn-Greene et al., 2007), and there is growing evidence that sleep disorders can trigger or aggravate a range of psychiatric conditions (Wehr et al., 1987; Ford and Kamerow, 1989; Breslau et al., 1996; Turek, 2005; Huang et al., 2007; Germain et al., 2008; Sateia, 2009). Schizophrenia is no exception. Sleep disturbances in schizophrenia have been described since Kraepelin (1919) and are associated with poorer coping skills and diminished quality of life (Goldman et al., 1996; Hofstetter et al., 2005). Subjective sleep disturbance is a common complaint throughout the course of schizophrenia (Lieberman et al., 2005), including in the prodrome (Miller et al., 2003). It is anecdotally associated with the initial onset of psychosis, and may serve as a predictor of psychotic decompensation in remitted patients (Benson, 2006). The presence of sleep disturbance in antipsychotic-naïve and unmedicated patients indicates that abnormal sleep is not merely a side-effect of medications, but instead may be a core feature of schizophrenia (for meta-analysis see Chouinard et al., 2004). In fact, antipsychotic medications often normalize sleep in schizophrenia (Krystal et al., 2008), and medication withdrawal has been associated with a progressive deterioration of sleep quality (Nofzinger et al., 1993), which, in turn, is associated with psychotic relapse (Dencker et al., 1986) and increased severity of positive symptoms (Chemerinski et al., 2002). Although disturbed sleep is a prominent feature of schizophrenia, the nature of the abnormality and its relations to the pathophysiology, signs, and symptoms of schizophrenia remain poorly understood.

Sleep deprivation is well-known to impair cognition and to alter associated patterns of brain activation in healthy individuals (Horne, 1993; Van Dongen et al., 2003; Chee and Chuah, 2008). Yet, in spite of its ubiquity in schizophrenia, disturbed sleep has been largely overlooked as a potential contributor to cognitive deficits. While there is a general tendency to regard disturbed sleep as secondary to the illness, this neglect may be exacerbated by difficulty specifying the exact nature of the disturbance, and from a lack of awareness of the critical role of sleep in cognition. In addition, although the sleep disturbance in schizophrenia is often sufficiently severe as to warrant independent clinical attention, it is seldom the primary complaint, and its potential contribution to cognitive deficits is unlikely to be considered. 
While cognitive deficits remain the strongest predictor of functional outcome in schizophrenia (Green et al., 2000), available antipsychotic medications are relatively ineffective in treating them (c.f., Sergi et al., 2007). Amelioration of cognitive deficits is a current priority of the schizophrenia research community, including government, academia, and industry, and is the focus of large-scale studies aimed at defining, characterizing, and quantifying these deficits for the purpose of evaluating the efficacy of interventions (e.g., Marder et al., 2004; Buchanan et al., 2007). A limitation of many of these efforts is that cognition is measured in cross-section. While this approach provides a valid snapshot of function, it does not capture critical aspects of learning and memory consolidation that happen offline, both over time and with sleep (e.g., Karni et al., 1998; Stickgold, 2005; Born et al., 2006; Brown and Robertson, 2007). Yet existing evidence, reviewed below, suggests that sleep-dependent processes are impaired in schizophrenia, and may contribute substantially to disability. We believe that understanding this contribution can open new and promising avenues for treatment.

There is now overwhelming evidence that initial memory encoding is followed by a prolonged period of consolidation, integration, and reorganization, that continues over days or even years (Schacter and Tulving, 1994). Much of this evolution of memories is mediated by sleep (Stickgold and Walker, 2007). It thus becomes important, when examining memory in schizophrenia, or in any neuropsychiatric disorder, to investigate deficits not only in initial encoding and recall, but also in time- and sleep-dependent memory processes. Even in cross-sectional measures of cognition, sleep should be considered as an important contributor to attention deficits that can blunt cognitive performance across domains, and are the most frequent and obvious casualty of poor sleep in healthy individuals. In this article, we will briefly review (i) what is known about abnormal sleep in schizophrenia, (ii) sleep-dependent memory consolidation in healthy individuals, (iii) recent findings of impaired sleep-dependent memory consolidation in schizophrenia, and (iv) implications of impaired sleep-dependent memory consolidation in schizophrenia. We conclude that abnormal sleep may contribute substantially to cognitive deficits in schizophrenia and should be a focus of study.

\section{WHAT IS KNOWN ABOUT ABNORMAL SLEEP IN SCHIZOPHRENIA?}

The most common subjective sleep complaints in schizophrenia are difficulty initiating and maintaining sleep (Chouinard et al., 2004; Benson, 2008; Cohrs, 2008). These complaints of insomnia have been verified by polysomnographic (PSG) recordings that show reduced sleep efficiency (total sleep time divided by time in bed), increased sleep onset latencies, and increased wake time after sleep onset (WASO), including in unmedicated and drug naïve patients (for meta-analyses see, Benca et al., 1992; Tandon et al., 1992; Lauer et al., 1997; Keshavan et al., 1998; Chouinard et al., 2004; Yang and Winkelman, 2006). Some studies also report alterations in circadian rhythms of hormone and activity patterns in schizophrenia (Rao et al., 1994; Martin et al., 2005) and increased rates of sleep disorders including sleep-related breathing disorders (e.g., obstructive sleep apnea), hypersomnolence, movement disorders (e.g., restless leg syndrome, periodic limb movement disorder), and parasomnias (for reviews see, Benson, 2006, 2008; Sateia, 2009).
PSG studies also report diverse abnormalities of sleep architecture (i.e., the amount and distribution of time spent in different sleep stages). Normal sleep in adult humans is divided into periods of rapid eye movement (REM) and non-rapid eye movement (NREM) sleep, with NREM sleep further divided into Stages 1-4 (Figure 1) (Rechtschaffen and Kales, 1968). Stages 3 and 4 are characterized by large $(>75 \mu \mathrm{V})$ delta $(0.5-2 \mathrm{~Hz})$ waves in the EEG, and together constitute "slow wave sleep" (SWS). (This nomenclature has recently changed, and NREM Stages 3 and 4 are now combined into one stage (Iber et al., 2007), but we defer to the older nomenclature, as it is used in all the literature reviewed here.) A typical night of sleep is made up of 4-5 REM cycles, each beginning with NREM sleep and ending with REM sleep, and lasting approximately $90 \mathrm{~min}$. While the cycle time is relatively constant across the night, most of the SWS occurs in the first half of the night, and most REM sleep occurs in the last half (Figure 1).

The most consistent electrophysiological sleep abnormality reported in schizophrenia is a decrease in the amount of SWS (Keshavan et al., 1990). Reductions have been reported in antipsychotic-naïve (Jus et al., 1968; Feinberg et al., 1969; Poulin et al., 2003), unmedicated (Keshavan et al., 1998; Yang and Winkelman, 2006), medicated (Goder et al., 2004), and remitted patients (Kupfer et al., 1970; Traub, 1972). Reduced SWS has also been observed in non-psychotic first-degree relatives of individuals with schizophrenia, suggesting that it is a trait associated with risk for schizophrenia (Keshavan et al., 2004).SWS reductions, however, are not consistently observed, even in antipsychotic-naïve patients (Tandon et al., 1992; Lauer et al., 1997) and have not survived metaanalysis (Chouinard et al., 2004). Caldwell and Domino (1967) reported that while $40 \%$ of patients showed almost a complete elimination of stage 4 SWS, the remaining $60 \%$ showed normal levels, suggesting that the reduction may characterize only a subset of patients. In addition, SWS changes are not specific to schizophrenia and are seen in major depression and other psychiatric disorders (Keshavan et al., 1990; Benca et al., 1992). Although abnormalities in REM sleep have also been reported, usually a decrease in REM latency (Tandon et al., 1992; Poulin et al., 2003) or an increase in REM density (Yang and Winkelman, 2006), meta-analyses have not revealed any systematic difference in REM sleep between patients and healthy or psychiatric controls (Benca et al., 1992; Chouinard et al., 2004). Both SWS and REM sleep abnormalities have been related to increased symptom severity, a literature that has been reviewed elsewhere (Benson, 2008).

Only a few studies have gone beyond sleep architecture to examine changes in the characteristics of the EEG power spectrum during sleep in schizophrenia. Slow wave activity and sleep spindles are

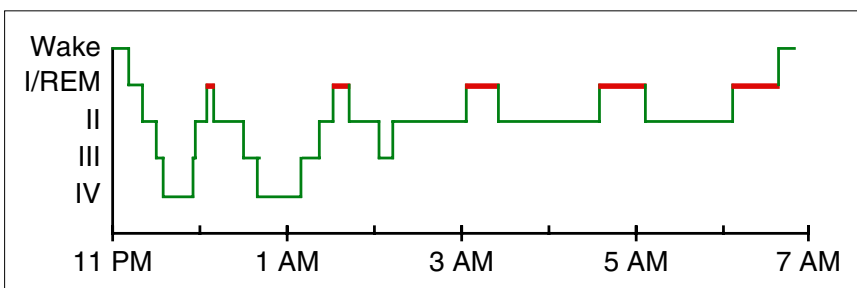

FIGURE 1 |A good night's sleep. The normal progression of sleep stages across a night of uninterrupted sleep. REM sleep is highlighted in red. 
particularly relevant here since slow wave activity has been correlated with overnight improvement of motor procedural learning (Huber et al., 2004) and accumulating evidence suggests that sleep spindles mediate sleep-dependent consolidation of both procedural (Walker et al., 2002; Fogel and Smith, 2006; Nishida and Walker, 2007; Peters et al., 2008; Rasch et al., 2008; Tamaki et al., 2008) and declarative (Clemens et al., 2005, 2006; Schabus et al., 2008) memory. Sleep spindles, a defining characteristic of stage 2 sleep, are brief powerful bursts of synchronous $12-15 \mathrm{~Hz}$ neuronal firing in thalamo-cortical networks, which reach peak density late in the night (De Gennaro et al., 2000). Spindles induce massive influxes of calcium ions into cortical pyramidal cells, which are believed to trigger intracellular, calcium-dependent mechanisms required for synaptic plasticity (Sejnowski and Destexhe, 2000). In schizophrenia, there have been reports of reduced slow wave activity (Hiatt et al., 1985; Keshavan et al., 1998; Goder et al., 2006) during SWS. There have also been mixed reports of changes in sleep spindles, including increased spindle counts (Hiatt et al., 1985), and both unchanged (Poulin et al., 2003) and reduced (Ferrarelli et al., 2007) sleep spindle density.

All of the usual suspects contribute to difficulties in characterizing sleep in schizophrenia. These include differences in sample size, demographic features, chronicity, and the definition and measurement of sleep parameters. Discrepant findings may also relate to the underlying pathophysiological and phenotypic heterogeneity of schizophrenia. Finally, by affecting neurotransmitter systems that play an important role in sleep regulation, treatments for schizophrenia, including antipsychotic, anticholinergic, and anti-adrenergic medications, have diverse effects on sleep (Monti and Monti, 2004; and for reviews see, Benson, 2008; Krystal et al., 2008; Kantrowitz et al., 2009). Thus, factors such as medication status (naive vs. unmedicated vs. medicated), duration, and type all contribute to variability in sleep measurements. While, overall, antipsychotic medications tend to improve measures of sleep maintenance and to normalize sleep architecture (e.g., Maixner et al., 1998; Salin-Pascual et al., 1999), their effects on the sleep processes that mediate memory consolidation are largely unknown.

\section{SLEEP-DEPENDENT MEMORY PROCESSING IN HEALTHY INDIVIDUALS}

The last decade has produced a wealth of evidence for the role of sleep in the offline processing of recent memories, describing sleep-dependent events at the molecular, cellular, neural network, regional brain activation, and behavioral levels, in birds (Dave et al., 1998), rodents (Wilson and McNaughton, 1994), cats (Frank et al., 2001), and humans (Stickgold and Walker, 2007). This evidence suggests an evolutionarily conserved function for sleep in the stabilization, enhancement, integration, and reorganization of a wide range of memory types, functions that we will collectively refer to as 'consolidation.' Most relevant to this review are the findings from human studies.

\section{PERCEPTUAL AND MOTOR PROCEDURAL LEARNING}

Procedural or skill learning is a category of non-declarative memory for how to perform various perceptual and motor tasks. Many visual (Stickgold et al., 2000), auditory (Gaab et al., 2004), motor sequence (Walker et al., 2002), and motor adaptation (Huber et al., 2004) tasks show improvements in performance after a night of sleep, but not after an equivalent period of daytime wake. Because there is an absolute improvement after sleep, the benefit cannot be simply ascribed to a passive protection against interference, or even to an active stabilization of the memories, as has been suggested for other types of learning. Rather, we and others have proposed that sleep gives rise to performance improvements that are either caused by or accompanied by systems level reorganizations of the initial memories that facilitate task automation (Atienza et al., 2004; Kuriyama et al., 2004; Fischer et al., 2005; Chee and Chuah, 2008). In this context, automation refers to a shift from controlled, effortful performance to performance that proceeds more efficiently (i.e., is faster, less variable, less vulnerable to interference, and has fewer errors), with reduced demands on attention (Shiffrin and Schneider, 1977) and a corresponding shift in the brain networks that support performance (Jueptner and Weiller, 1998). While automation is known to develop with practice, there is now direct evidence that automation can also develop with sleep.

Overnight improvement on a procedural visual discrimination task correlates with SWS early in the night and REM sleep late in the night (Stickgold et al., 2000), with SWS appearing to stabilize the original memory and REM sleep subsequently enhancing it (Mednick et al., 2003). In contrast, improvement on a procedural motor sequence tasks correlates with Stage 2 NREM sleep late in the night (Walker et al., 2002). In this case, improvement is characterized by faster, more accurate and more uniform transitions within the sequence, suggesting automation of the motor program (Kuriyama et al., 2004; Fischer et al., 2005). These skill enhancements are accompanied by changes in task-related brain activation patterns, including reduced activation in regions such as prefrontal cortex that mediate the conscious monitoring of performance (Fischer et al., 2005; Walker et al., 2005). Evidence for sleep-dependent automation is also found in a study of auditory learning in which participants learned to detect a deviant tone in a complex tone sequence (Atienza et al., 2004), although sleep stage correlates of this automation were not determined. Sleepdependent changes in the 'mismatch negativity' (MMN) and P3a event-related potentials generated by deviant tones suggest that sleep reduced the voluntary attentional effort required for successful stimulus discrimination.

\section{VERBAL MEMORY}

Verbal declarative memory also benefits from post-training sleep. Performance on a word-pairs cued recall task has consistently been found to be better after a night of sleep than after an equal period of daytime wake (Plihal and Born, 1997; Ellenbogen et al., 2009), and to show greater resistance to subsequent interference after sleep (Ellenbogen et al., 2006, 2009). At least some of these benefits correlate with times of night rich in SWS (Plihal and Born, 1997). Sleep also facilitates the selective retention of gist memory for word lists, resulting the next morning in a less accurate, but arguably more useful abstract memory, with enhanced gist and reduced detail (Payne et al., 2006). Interestingly, when gist is enhanced, improved memory recall correlates with decreases in SWS.

\section{EMOTIONAL MEMORY}

Declarative memory for emotional words and pictures is also enhanced by sleep. When individuals studied pictures with 
either neutral or aversive objects placed on neutral backgrounds, subsequent recognition of the aversive objects, relative to their backgrounds, was enhanced after sleep compared to wake (Payne et al., 2008). In contrast, sleep offered no benefit for memory of neutral objects or their backgrounds. Thus, sleep seems to unbind complex scenes and selectively enhance memory for the emotional components.

\section{COMPLEX COGNITIVE PROCEDURAL LEARNING}

In addition to benefiting simple procedural and declarative memories, sleep can also produce striking benefits for more complex forms of learning, such as rule extraction and insight. Using the remote associates task (Mednick, 1962), Cai et al. (2009) reported that napping facilitates discovery of the target word that links three otherwise unrelated words together (e.g., "heart", "sixteen", and "cookie" are linked by the word "sweet"), a benefit that correlates with the amount of REM sleep obtained after initial exposure to the word triads. Similarly, Wagner et al. (2004) have shown than sleep enhances insight into a more efficient method of solving a class of mathematical problems. In these and other instances (e.g., Ellenbogen et al., 2007), sleep facilitates the identification of associations and commonalities that are less easily discovered during wake. Interestingly, in some cases this identification leads to subsequent conscious awareness of the new information (e.g., Wagner et al., 2004; Cai et al., 2009), while in other cases improved performance develops without any conscious awareness (e.g., Djonlagic et al., 2005; Ellenbogen et al., 2007).

Looking across memory paradigms, it appear likely that individual sleep stages correlate not with types of memory, but rather with stages in the "consolidation" process. Overall, SWS appears to correlate with stabilization of memories, possibly through synaptic level processes that reinforce the memory in the form in which it was originally encoded. In contrast, REM sleep, and possibly Stage 2 NREM, appear to lead to systems level reorganization of memories, resulting in their enhancement, automation, and integration into larger associative networks.

\section{IMPAIRED SLEEP-DEPENDENT MEMORY CONSOLIDATION IN SCHIZOPHRENIA}

There are still relatively few investigations of the role of sleep in cognition in schizophrenia. One line of research suggests that impaired sleep exacerbates attention deficits. An early study showed that among unmedicated chronic schizophrenia patients $(n=10)$, those who made more errors of omission on a continuous performance test had significantly less SWS (Orzack et al., 1977). Similarly, Forest et al. (2007) reported that increased reaction time on tests of attention in antipsychotic-naïve patients with schizophrenia $(n=8)$ and healthy controls $(n=8)$ correlated with decreased sleep spindle density in both groups, and with Stage 4 SWS sleep duration only in schizophrenia. Finally, in a sample of chronic patients who had been withdrawn from their medications $(n=15)$, Yang and Winkelman (2006) clinical ratings of cognitive symptoms correlated with both decreased SWS and decreased REM density.

A second line of research suggests that impaired sleep in schizophrenia also leads to deficits in sleep-dependent memory consolidation and automation. In medicated schizophrenia patients $(n=17)$, reductions in SWS and sleep efficiency correlated with reduced recall of the Rey-Osterrieth Complex Figure, a test of visuospatial memory, following a night of sleep (Goder et al., 2004). In an exploratory study, Goder et al. (2006) found that slow wave activity in NREM sleep positively correlated with both better performance and overnight improvement on a range of neuropsychological measures in healthy controls. In contrast, medicated schizophrenia patients $(n=16)$, who showed reduced slow wave activity, showed relatively few significant correlations with slow wave activity, and these were evenly split between positive and negative correlations. In a recent study of chronic patients taking amisulpride ( $n=26)$, Goder et al. (2008) reported that patients with more SWS had better recognition memory for words that were learned prior to sleep, as did those with a higher sleep spindle density.

Further evidence of impaired sleep-dependent memory consolidation in schizophrenia comes from two studies of procedural memory, one behavioral (Manoach et al., 2004) and one that included a night of PSG recording (Manoach et al., 2009), which used a finger tapping motor sequence task (MST, Karni et al., 1998) with chronic medicated patients with schizophrenia (Study 1: $n=20$; Study 2: $n=14$ ). In spite of intact practice-dependent learning during training, neither study found evidence of the subsequent sleep-dependent improvement in performance that was seen in healthy controls (Figure 2). The absence of significant overnight improvement in patients in the second study (Manoach et al., 2009) occurred in the context of no differences from controls in the amounts or distribution of time spent in specific sleep stages, or in any index of awakenings.

In healthy young adults, overnight improvement on the MST and other simple procedural motor skill tasks correlates with the amount of stage 2 NREM sleep in the last quarter of the night (S2q4, Smith and MacNeill, 1994; Walker et al., 2002; Fogel et al., 2007). MST improvement also correlates with the number and density of fast spindles (Rasch et al., 2008) as well as with an asymmetry of spindle density and power at central electrodes (right C4 $>$ left $\mathrm{C} 3$ ), which may reflect selective changes in the right motor cortex related to learning since it was the left hand that performed the task (C4-C3, Nishida and Walker, 2007). In this context, it is striking that, compared to healthy controls, schizophrenia patients showed a significant $45 \%$ reduction in fast sigma frequency power, and $43 \%$ reduction in spindle density, at C4 during S2q4 sleep following MST training (Manoach et al., 2009).

Although patients with schizophrenia did not show significant overnight improvement as a group, there was considerable variability in the amount of improvement, which correlated with the amount of time spent in specific sleep stages (Manoach et al., 2009). As in young healthy individuals (Walker et al., 2002), time spent in S2q4 sleep predicted overnight improvement (Figure 3A), but so did SWS duration (Figure 3B), an effect not previously seen with the MST. When the product of SWS and S2q4 sleep was added to a regression model, their individual contributions were no longer significant, and only their product was, accounting for $77 \%$ of the variance in overnight improvement in schizophrenia (Figure 3C). This suggests that both SWS and S2q4 sleep are necessary for consolidation and is consistent with a two-stage model of procedural memory consolidation (Stickgold et al., 2000).

In a prior study of healthy individuals, overnight improvement on a visuoperceptual procedural learning task correlated with both 


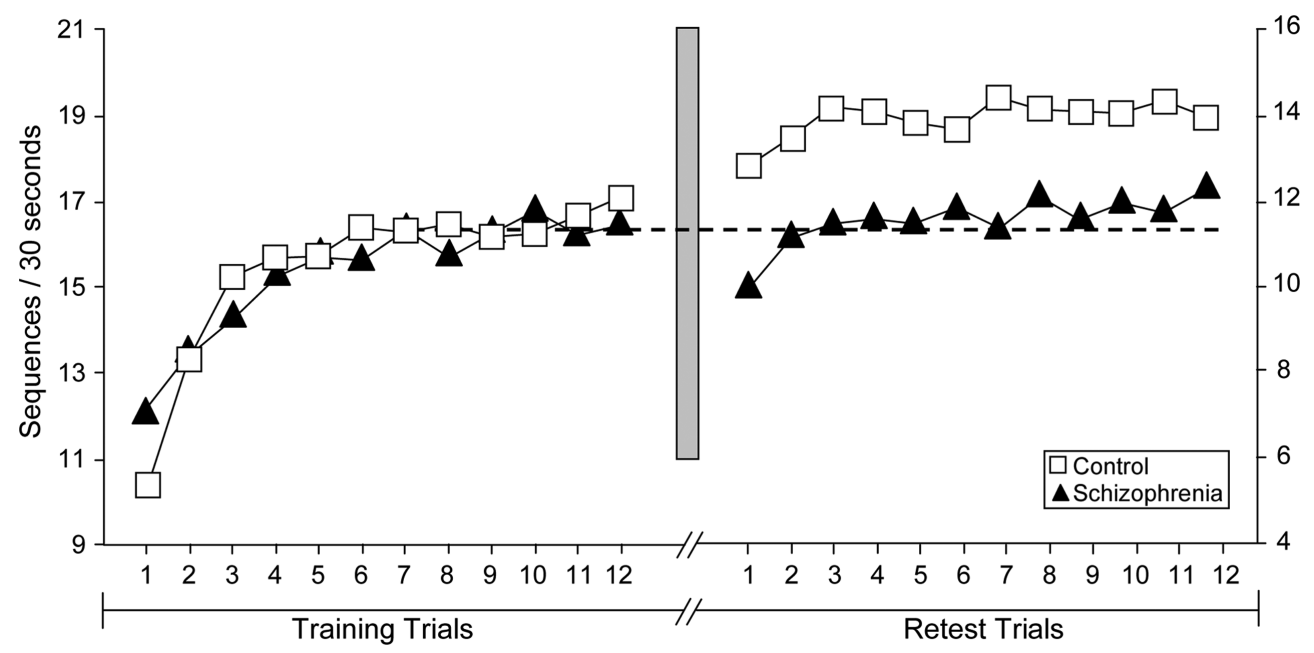

FIGURE 2 | Motor skill learning across training and test trials of the motor sequence task (MST) for healthy control participants ( $n=14$, open squares) and schizophrenia patients ( $n=20$, closed triangles) from Manoach et al. (2004). The data point for each trial represents the group average. The $y$-axes represent the number of correct sequences typed in each 30-s epoch. Note that the $y$-axes are scaled separately for controls (left) and patients (right) to better illustrate the qualitative similarity of learning curves on Day 1 and the failure of overnight improvement in the schizophrenia group only. The dashed line is positioned at the mean value of the last three training trials for both the control and patient groups. The shaded bar represents the passage of $24 \mathrm{~h}$, including a night of sleep. Patients and controls did not differ in the amount of learning during training, but only controls showed significant overnight improvement.
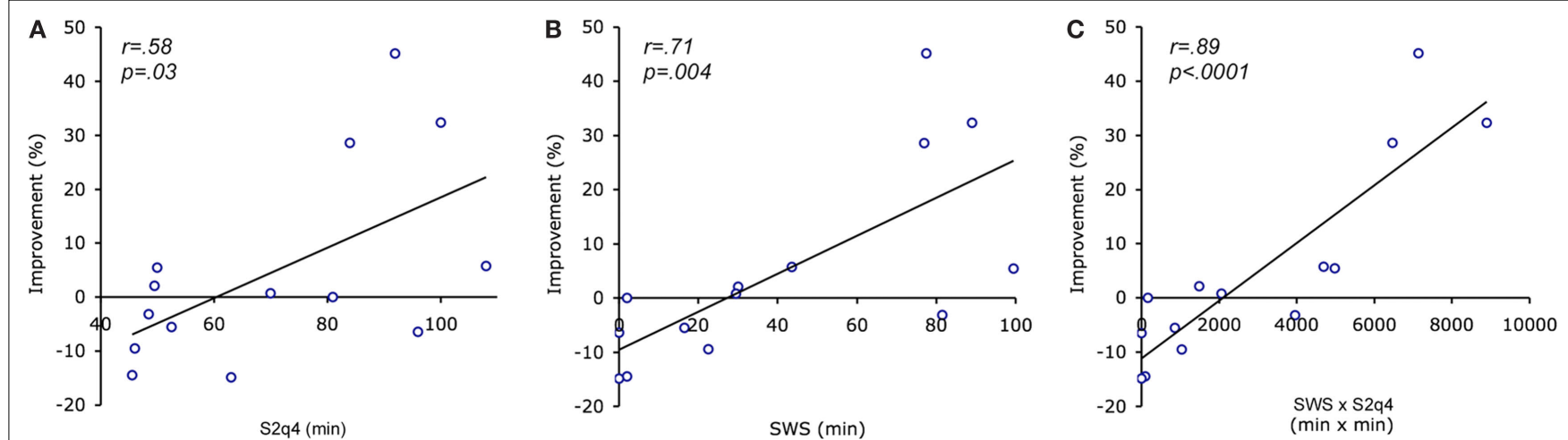

FIGURE 3 | Correlation of overnight improvement with minutes spent in slow wave sleep (SWS) and stage 2 sleep in the last quartile of the night (S2q4) in schizophrenia patients. (A) Correlation with S2q4 sleep; (B) Correlation with SWS; (C) Correlation with the product of SWS and S2q4 sleep (SWS x S2q4).

SWS early in the night and REM sleep in the last quarter, but even more strongly with their product (Stickgold et al., 2000). A subsequent study of naps showed that while naps containing SWS prevented deterioration in visuoperceptual performance over the day, naps with both SWS and REM sleep led to same-day improvement (Mednick et al., 2002). These findings suggest that SWS, which is predominant early in the night, stabilizes visuoperceptual procedural memory, while REM sleep later in the night enhances it. A similar model fits our motor procedural memory consolidation findings in schizophrenia (Manoach et al., 2009). While both SWS and S2q4 sleep correlated with improvement measured at the start of the test session, when this initial improvement was broken into its component parts, a striking double dissociation was seen. SWS appeared to prevent the delayed expression of improvement, which characterizes MST performance in healthy middle-aged (Manoach et al., 2004) and elderly participants (McKinley, 2008), while S2q4 sleep appeared to facilitate the sleep-dependent enhancement seen after this delay.

These findings provide direct evidence of a deficit in sleepdependent memory consolidation in medicated patients with chronic schizophrenia. That this deficit occurred in the context of normal sleep architecture suggests that it is not the amount or distribution of time spent in different sleep stages that is culpable, but rather specific memory consolidation processes that are normally activated during sleep (Manoach et al., 2004), particularly processes occurring during SWS and S2q4 sleep. An important caveat is that the samples in these two studies were too small and the medications, which were clinically determined, were too diverse to adequately evaluate medication effects. Although antipsychotic dose, as measured by chlorpromazine equivalent, was not correlated with any 
measure of overnight improvement, a study of medication-naïve patients would be required to determine the extent to which deficits in sleep-dependent memory consolidation reflect medication side-effects versus a disease process.

\section{IMPLICATIONS OF IMPAIRED SLEEP-DEPENDENT MEMORY CONSOLIDATION IN SCHIZOPHRENIA}

Sleep facilitates a wide range of processes that mediate the evolution of memories over time. These sleep-dependent processes aid in the stabilization, strengthening, integration, and reorganization of memories to increase their durability, flexibility, and automation. If all sleep-dependent memory processing were lacking, we suspect that this would impair not only the fine-tuning of complex skills and memories, but would also lead to profound difficulties in carrying out the basic activities of daily life.

There are still very few studies of the role of sleep in the cognitive deficits of schizophrenia. These early studies have small samples and many simply correlate sleep measurements with cross-sectional measures of neuropsychological performance, suggesting that sleep affects cognitive performance, primarily attention, in schizophrenia, as it does in healthy individuals. The few studies that evaluated the role of sleep in memory consolidation by comparing performance prior to and following a night of sleep have documented reduced overnight improvement in patients as a group, even in the context of comparable sleep architecture. Unfortunately, these studies have only included medicated patients. But regardless of the underlying mechanisms and whether they reflect treatment or disease process, findings of impaired sleep-dependent memory consolidation have important implications for understanding and treating cognitive dysfunction in schizophrenia.

We propose that the observed failures of sleep-dependent memory consolidation represent a breakdown, not in the overall structure of sleep, but rather in specific memory consolidation processes that are normally activated during sleep. These sleepdependent memory processes normally lead to task automation,

\section{REFERENCES}

Atienza, M., Cantero, J. L., and Stickgold, R. (2004). Posttraining sleep enhances automaticity in perceptual discrimination. J. Cogn. Neurosci. 16, 53-64.

Benca, R. M. (1996). Sleep in psychiatric disorders. Neurol. Clin. 14, 739-764.

Benca, R. M., Obermeyer, W. H., Thisted, R. A., and Gillin, J. C. (1992). Sleep and psychiatric disorders. A meta-analysis. Arch. Gen. Psychiatry 49, 651-668; discussion 669-670.

Benson, K. L. (2006). Sleep in schizophrenia: impairments, correlates, and treatment. Psychiatr. Clin. North Am. 29, 1033-1045; abstract ix-x.

Benson, K. L. (2008). Sleep in schizophrenia. Sleep Med. Clin. 3, 251-260.

Born, J., Rasch, B., and Gais, S. (2006). Sleep to remember. Neuroscientist 12, 410-424.

Breslau, N., Roth, T., Rosenthal, L., and Andreski, P. (1996). Sleep disturbance

resulting in performance that is faster, less variable, and less dependent on voluntary attention (Atienza et al., 2004; Kuriyama et al., 2004) and to more efficient patterns of brain activation, particularly in the prefrontal cortex (Fischer et al., 2005; Walker et al., 2005; Chee and Chuah, 2008). These recent findings of reduced sleep-dependent memory consolidation support the hypothesis of deficient automation in schizophrenia (Granholm et al., 1991; Manoach et al., 2000; Manoach, 2003) and extend this hypothesis to sleep-dependent processes. A fundamental breakdown in sleepdependent automation in schizophrenia would make it necessary to allocate limited-capacity attentional resources to task elements that, in healthy individuals, have been automated as a function of sleep. This would leave fewer resources available for higher-order task demands that require cognitive control. It is this interaction between automatic and controlled processes that normally allows a limited capacity brain to carry out complex cognitive tasks. An impairment in sleep dependent automation could contribute substantially to the generalized cognitive deficits that are a hallmark of schizophrenia (Chapman and Chapman, 1978; Dickinson and Harvey, 2009).

\section{CONCLUDING REMARKS}

Sleep disturbances are common in schizophrenia. From a purely clinical perspective, they should be vigorously treated since in schizophrenia, like in other psychiatric disorders (Fava et al., 2006; Manber et al., 2008), treating sleep may also improve symptoms. The existing literature, reviewed here, suggests that treating sleep may also improve cognition in schizophrenia. But to identify targets for treatment, it is important to understand the mechanisms underlying normal sleep-dependent memory consolidation, how these are altered in schizophrenia, and how they are affected by medications. Understanding the contribution of sleep to cognitive deficits, and clarifying the role of medications in mediating these effects, may open new avenues to ameliorating cognitive dysfunction and thereby improve outcome in schizophrenia.

movement patterns during sleep in chronic schizophrenic patients. Electroencephalogr. Clin. Neurophysiol. 22, 414-420.

Chapman, L. J., and Chapman, J.P. (1978). The measurement of differential deficit. J. Psychiatr. Res. 14, 303-311.

Chee, M. W., and Chuah, L. Y. (2008). Functional neuroimaging insights into how sleep and sleep deprivation affect memory and cognition. Curr. Opin. Neurol. 21, 417-423.

Chemerinski, E., Ho, B. C., Flaum, M. Arndt, S., Fleming, F., and Andreasen, N. C. (2002). Insomnia as a predictor for symptom worsening following antipsychotic withdrawal in schizophrenia. Compr. Psychiatry 43, 393-396.

Chouinard, S., Poulin, J., Stip, E., and Godbout, R. (2004). Sleep in untreated patients with schizophrenia: a meta-analysis. Schizophr. Bull. 30, 957-967.
Clemens, Z., Fabo, D., and Halasz, P. (2005) Overnight verbal memory retention correlates with the number of sleep spindles. Neuroscience 132, 529-535.

Clemens, Z., Fabo, D., and Halasz, P. (2006). Twenty-four hours retention of visuospatial memory correlates with the number of parietal sleep spindles. Neurosci. Lett. 403, 52-56.

Cohrs, S. (2008). Sleep disturbances in patients with schizophrenia: impact and effect of antipsychotics. CNS Drugs 22, 939-962.

Dave, A. S., Yu, A. C., and Margoliash, D. (1998). Behavioral state modulation of auditory activity in a vocal motor system. Science 282, 2250-2254.

De Gennaro, L., Ferrara, M., and Bertini, M. (2000). Topographical distribution of spindles: variations between and within nrem sleep cycles. Sleep Res. Online 3, 155-160.

Dencker, S. J., Malm, U., and Lepp, M. (1986). Schizophrenic relapse after 
drug withdrawal is predictable. Acta Psychiatr. Scand. 73, 181-185.

Dickinson, D., and Harvey, P. D. (2009). Systemic hypotheses for generalized cognitive deficits in schizophrenia: a new take on an old problem. Schizophr. Bull. 35, 403-414.

Djonlagic, I., Rosenfeld, A., and Stickgold, R. (2005). Sleep-dependent consolidation of category learning. Sleep 28, A348.

Ellenbogen, J. M., Hu, P. T., Payne, J. D., Titone, D., and Walker, M. P. (2007). Human relational memory requires time and sleep. Proc. Natl. Acad. Sci. U.S.A. 104, 7723-7728.

Ellenbogen, J. M., Hulbert, J., Jiang, Y., and Stickgold, R. (2009). The sleeping brain's influence on verbal memory: boosting resistance to interference. PLoS One.4, e4117. doi: 10.1371/journal.pone.0004117

Ellenbogen, J. M., Hulbert, J. C., Stickgold, R., Dinges, D. F., and Thompson-Schill, S. L. (2006). Interfering with theories of sleep and memory: sleep, declarative memory, and associative interference. Curr. Biol. 16, 1290-1294.

Fava, M., McCall, W. V., Krystal, A., Wessel, T., Rubens, R., Caron, J., Amato, D., and Roth, T. (2006). Eszopiclone co-administered with fluoxetine in patients with insomnia coexisting with major depressive disorder. Biol. Psychiatry 59, 1052-1060.

Feinberg, I., Braun, M., Koresko, R. L., and Gottlieb, F. (1969). Stage 4 sleep in schizophrenia. Arch Gen. Psychiatry 21, 262-266.

Ferrarelli, F., Huber, R., Peterson, M. J., Massimini, M., Murphy, M., Riedner, B. A., Watson, A., Bria, P., and Tononi, G. (2007). Reduced sleep spindle activity in schizophrenia patients. Am. J. Psychiatry 164, 483-492.

Fischer, S., Nitschke, M. F., Melchert, U. H., Erdmann, C., and Born,J. (2005). Motor memory consolidation in sleep shapes more effective neuronal representations. J. Neurosci. 25, 11248-11255.

Fogel, S. M., and Smith, C. T. (2006). Learning-dependent changes in sleep spindles and Stage 2 sleep. J. Sleep Res. $15,250-255$.

Fogel, S. M., Smith, C. T., and Cote, K. A. (2007). Dissociable learningdependent changes in REM and non-REM sleep in declarative and procedural memory systems. Behav. Brain Res. 180, 48-61.

Ford, D. E., and Kamerow, D. B. (1989). Epidemiologic study of sleep disturbances and psychiatric disorders. An opportunity for prevention? JAMA 262, 1479-1484.

Forest, G., Poulin, J., Daoust, A. M., Lussier, I., Stip, E., and Godbout, R.
(2007). Attention and non-REM sleep in neuroleptic-naive persons with schizophrenia and control participants. Psychiatry Res. 149, 33-40.

Frank, M. G., Issa, N. P., and Stryker, M. P. (2001). Sleep enhances plasticity in the developing visual cortex. Neuron 30 , 275-287.

Gaab, N., Paetzold, M., Becker, M., Walker, M. P., and Schlaug, G. (2004). The influence of sleep on auditory learning: a behavioral study. Neuroreport 15, 731-734.

Germain,A.,Buysse, D.J., and Nofzinger, E. (2008). Sleep-specific mechanisms underlying posttraumatic stress disorder: integrative review and neurobiological hypotheses. Sleep Med. Rev. $12,185-195$.

Goder, R., Aldenhoff, J. B., Boigs, M., Braun, S., Koch, J., and Fritzer, G. (2006). Delta power in sleep in relation to neuropsychological performance in healthy subjects and schizophrenia patients. J. Neuropsychiatry Clin. Neurosci. 18, 529-535.

Goder, R., Boigs, M., Braun, S., Friege, L., Fritzer, G., Aldenhoff, J. B., and Hinze-Selch, D. (2004). Impairment of visuospatial memory is associated with decreased slow wave sleep in schizophrenia. J. Psychiatr. Res. 38, 591-599.

Goder, R., Fritzer, G., Gottwald, B., Lippmann, B., Seeck-Hirschner, M., Serafin, I., and Aldenhoff, J. B. (2008). Effects of olanzapine on slow wave sleep, sleep spindles and sleeprelated memory consolidation in schizophrenia. Pharmacopsychiatry 41, 92-99.

Goldman,M., Tandon, R.,DeQuardo, J. R., Taylor, S. F., Goodson, J., and McGrath, M. (1996). Biological predictors of 1-year outcome in schizophrenia in males and females. Schizophr. Res. 21, 65-73.

Granholm, E., Asarnow, R. F., and Marder, S. R. (1991). Controlled information processing resources and the development of automatic detection responses in schizophrenia. J. Abnorm. Psychol. 100, 22-30.

Green, M. F., Kern, R. S., Braff, D. L., and Mintz, J. (2000). Neurocognitive deficits and functional outcome in schizophrenia: are we measuring the "right stuff"? Schizophr. Bull. 26, 119-136.

Hiatt, J. F., Floyd, T. C., Katz, P. H., and Feinberg, I. (1985). Further evidence of abnormal non-rapid-eye-movement sleep in schizophrenia. Arch Gen. Psychiatry. 42, 797-802.

Hofstetter, J. R., Lysaker, P. H., and Mayeda, A. R. (2005). Quality of sleep in patients with schizophrenia is associated with quality of life and coping. $B M C$ Psychiatry 5, 13.
Horne, J. A. (1993). Human sleep, sleep loss and behaviour. Implications for the prefrontal cortex and psychiatric disorder. Br. J. Psychiatry 162, 413-419.

Huang, Y. S., Guilleminault, C., Li, H. Y., Yang, C. M., Wu, Y. Y., and Chen, N. H. (2007).Attention-deficit/hyperactivity disorder with obstructive sleep apnea: a treatment outcome study. Sleep Med. $8,18-30$.

Huber, R., Ghilardi, M. F., Massimini, M., and Tononi, G. (2004). Local sleep and learning. Nature 430, 78-81.

Iber, C., Ancoli-Israel, S., Chesson, A. L. and Quan, S. F. (2007). The AASM Manual for the Scoring of Sleep and Associated Events: Rules, Terminology, and Technical Specifications. Westchester, IL, American Academy of Sleep Medicine.

Jueptner, M., and Weiller, C. (1998). A review of differences between basal ganglia and cerebellar control of movements as revealed by functional imaging studies. Brain 121, 1437-1449.

Jus, K., Kiljan, A., Wilczak, H., Kubacki, A., Rzepecki, J., and Jus, A. (1968) [Polygraphic study of night sleep in schizophrenia before and during treatment with phenothiazine derivatives]. Ann. Med. Psychol. (Paris) 126, 713-725.

Kahn-Greene, E. T., Killgore, D. B. Kamimori, G. H., Balkin, T. J., and Killgore, W. D. (2007). The effects of sleep deprivation on symptoms of psychopathology in healthy adults. Sleep Med. 8, 215-221.

Kantrowitz, J., Citrome, L., and Javitt, D. (2009). GABA(B) receptors, schizophrenia and sleep dysfunction: a review of the relationship and its potential clinical and therapeutic implications. CNS Drugs 23, 681-691.

Karni, A., Meyer, G., Rey-Hipolito, C., Jezzard, P., Adams, M. M., Turner, R., and Ungerleider, L. G. (1998). The acquisition of skilled motor performance: fast and slow experiencedriven changes in primary motor cortex. Proc. Natl. Acad. Sci. U.S.A. 95, 861-868.

Keshavan, M. S., Diwadkar, V. A., Montrose, D. M., Stanley, J. A., and Pettegrew, J. W. (2004). Premorbid characterization in schizophrenia: the Pittsburgh High Risk Study. World Psychiatry 3, 163-168.

Keshavan, M. S., Reynolds, C. F., and Kupfer, D. J. (1990). Electroencephalographic sleep in schizophrenia: a critical review. Compr. Psychiatry 31, 34-47.

Keshavan, M. S., Reynolds, C. F., 3rd, Miewald, M. J., Montrose, D. M., Sweeney, J. A., Vasko, R. C., Jr., and Kupfer, D. J. (1998). Delta sleep deficits in schizophrenia: evidence from automated analyses of sleep data. Arch Gen. Psychiatry. 55, 443-448.

Kraepelin, E. (1919). Dementia praecox and paraphrenia. Edinburgh, E.S. Livingston.

Krystal,A.D., Goforth, H.W., and Roth, T. (2008). Effects of antipsychotic medications on sleep in schizophrenia. Int. Clin. Psychopharmacol. 23, 150-160.

Kupfer, D. J., Wyatt, R. J., Scott, J., and Snyder, F. (1970). Sleep disturbance in acute schizophrenic patients. Am. J. Psychiatry 126, 1213-1223.

Kuriyama, K., Stickgold, R., and Walker, M. P. (2004).Sleep-dependent learning and motor-skill complexity. Learn. Mem. 11, 705-713.

Lauer, C. J., Schreiber, W., Pollmacher, T., Holsboer, F., and Krieg, J. C. (1997). Sleep in schizophrenia: a polysomnographic study on drug-naive patients. Neuropsychopharmacology $16,51-60$.

Lieberman, J.A.,Stroup, T.S., McEvoy, J. P., Swartz, M. S., Rosenheck, R. A., Perkins, D. O., Keefe, R. S., Davis, S. M., Davis, C. E., Lebowitz, B. D., Severe, J., and Hsiao, J. K. (2005). Effectiveness of antipsychotic drugs in patients with chronic schizophrenia. N. Engl. J. Med. 353, 1209-1223.

Maixner, S., Tandon, R., Eiser,A., Taylor, S., DeQuardo, J.R., and Shipley, J. (1998). Effects of antipsychotic treatment on polysomnographic measures in schizophrenia: a replication and extension Am. J. Psychiatry 155, 1600-1602.

Manber, R., Edinger, J. D., Gress, J. L., San Pedro-Salcedo, M. G., Kuo, T. F., and Kalista, T. (2008). Cognitive behavioral therapy for insomnia enhances depression outcome in patients with comorbid major depressive disorder and insomnia. Sleep 31, 489-495.

Manoach, D. S. (2003). Prefrontal cortex dysfunction during working memory performance in schizophrenia: reconciling discrepant findings. Schizophr. Res. 60, 285-298.

Manoach, D. S., Cain, M. S., Vangel, M. G., Khurana, A., Goff, D. C., and Stickgold, R. (2004). A failure of sleep-dependent procedural learning in chronic, medicated schizophrenia. Biol. Psychiatry 56, 951-956.

Manoach,D.S., Gollub, R. L., Benson, E. S., Searl, M. M., Goff, D. C., Halpern, E., Saper, C. B., and Rauch, S. L. (2000). Schizophrenic subjects show aberrant fMRI activation of dorsolateral prefrontal cortex and basal ganglia during working memory performance. Biol. Psychiatry 48, 99-109.

Manoach, D. S., Thakkar, K. N., Stroynowski, E., Ely, A., McKinley, S. K., Wamsley, E., Djonlagic, I., Vangel, M. G., Goff, D. C., and Stickgold, R. (2009). Reduced overnight consolidation 
of procedural learning in chronic medicated schizophrenia is related to specific sleep stages. J. Psychiatr. Res.

Marder, S. R., Fenton, W., and Youens, K. (2004). Schizophrenia, IX: cognition in schizophrenia - the MATRICS initiative. Am. J. Psychiatry 161, 25.

Martin,J. L., Jeste, D.V., and Ancoli-Israel, S. (2005). Older schizophrenia patients have more disrupted sleep and circadian rhythms than age-matched comparison subjects. J. Psychiatr. Res. 39, 251-259.

McKinley, S. K. (2008). Sleep, learning, and memory in the elderly, Neurobiology Cambridge, Harvard University.

Mednick, S., Nakayama, K., and Stickgold, R. (2003). Sleep-dependent learning: a nap is as good as a night. Nat. Neurosci. 6, 697-698.

Mednick, S. A. (1962). The associative basis of the creative process. Psychol. Rev. 69, 220-232.

Mednick,S.C.,Nakayama, K.,Cantero, J. L., Atienza, M., Levin, A. A., Pathak, N., and Stickgold, R. (2002). The restorative effect of naps on perceptual deterioration. Nat. Neurosci. 5, 677-681.

Miller, T. J., Zipursky, R. B., Perkins, D., Addington, J., Woods, S. W., Hawkins, K.A., Hoffman, R., Preda, A., Epstein, I., Addington, D., Lindborg, S., Marquez,E., Tohen, M., Breier,A., and McGlashan, T. H. (2003). The PRIME North America randomized doubleblind clinical trial of olanzapine versus placebo in patients at risk of being prodromally symptomatic for psychosis. II. Baseline characteristics of the "prodromal" sample. Schizophr. Res. 61, 19-30.

Monti, J. M., and Monti, D. (2004). Sleep in schizophrenia patients and the effects of antipsychotic drugs. Sleep Med. Rev. 8, 133-148.

Nishida, M., and Walker, M. P. (2007). Daytime naps, motor memory consolidation and regionally specific sleep spindles. PLoS One 2, e341. doi: 10.1371/journal.pone.0000341.

Nofzinger, E. A., van Kammen, D. P., Gilbertson, M. W., Gurklis, J. A., and Peters, J. L. (1993). Electroencephalographic sleep in clinically stable schizophrenic patients: two-weeksversussix-weeks neurolepticfree. Biol. Psychiatry 33, 829-835.

Orzack, M. H., Hartmann, E. L., and Kornetsky, C. (1977). The relationship between attention and slow-wave sleep in chronic schizophrenia [proceedings]. Psychopharmacol. Bull. 13, 59-61.
Payne, J. D., Propper, R., Walker, M. P., and Stickgold, R. (2006). Sleep increases false recall of semantically related words in the Deese-Roediger-McDermott memory task. Sleep 29, A373.

Payne, J. D., Stickgold, R., Swanberg, K., and Kensinger, E.A. (2008). Sleep preferentially enhances memory for emotional components of scenes. Psychol. Sci. 19, 781-788.

Peters, K. R., Ray, L., Smith, V., and Smith, C. (2008). Changes in the density of stage 2 sleep spindles following motor learning in young and older adults. J. Sleep Res. 17, 23-33.

Plihal, W., and Born, J. (1997). Effects of early and late nocturnal sleep on declarative and procedural memory. J. Cogn. Neurosci. 9, 534-547.

Poulin, J., Daoust, A. M., Forest, G., Stip, E., and Godbout, R. (2003). Sleep architecture and its clinical correlates in first episode and neuroleptic-naive patients with schizophrenia. Schizophr. Res. 62, 147-153.

Rao, M.L., Gross, G., Strebel, B., Halaris, A., Huber, G., Braunig, P., and Marler, M. (1994). Circadian rhythm of tryptophan, serotonin, melatonin, and pituitary hormones in schizophrenia. Biol. Psychiatry 35, 151-163.

Rasch, B., Pommer, J., Diekelmann, S., and Born, J. (2008). Pharmacological REM sleep suppression paradoxically improves rather than impairs skill memory. Nat. Neurosci. 12, 396-397.

Rechtschaffen, A., and Kales, A. (ed.) (1968). A manual standardized terminology, techniques and scoring system for sleep stages of human subjects. US Department of Health, Bethesda, MD.

Salin-Pascual, R. J., HerreraEstrella, M., Galicia-Polo, L., and Laurrabaquio, M. R. (1999). Olanzapine acute administration in schizophrenic patients increases delta sleep and sleep efficiency. Biol. Psychiatry 46, 141-143.

Sateia, M. J. (2009). Update on sleep and psychiatric disorders. Chest 135, 1370-1379.

Schabus, M., Hoedlmoser, K., Pecherstorfer, T., Anderer, P., Gruber, G., Parapatics, S., Sauter, C., Kloesch, G., Klimesch, W., Saletu, B., and Zeitlhofer, J. (2008). Interindividual sleep spindle differences and their relation to learningrelated enhancements. Brain Res. 1191, 127-135.
Schacter, D. L., and Tulving, E. (1994). What are the memory systems of 1994. In Memory Systems 1994, D. L. Schacter and E. Tulving, eds (Cambridge, MA, The MIT Press), pp. 1-38.

Sejnowski, T. J., and Destexhe, A. (2000). Why do we sleep? Brain Res. 886 , 208-223.

Sergi, M. J., Green, M. F., Widmark, C., Reist, C., Erhart, S., Braff, D. L., Kee, K. S., Marder, S. R., and Mintz, J. (2007). Social cognition [corrected] and neurocognition: effects of risperidone, olanzapine, and haloperidol. Am. J. Psychiatry 164, 1585-1592.

Shiffrin, R. M., and Schneider, W. (1977). Controlled and automatic human information processing. II. Perceptual learning, automatic attending, and a general theory. Psychol. Rev. 84, 127-190.

Smith, C., and MacNeill, C. (1994). Impaired motor memory for a pursuit rotor task following Stage 2 sleep loss in college students. J. Sleep Res. 3, 206-213.

Stickgold, R. (2005). Sleep-dependent memory consolidation. Nature 437, 1272-1278.

Stickgold, R., and Walker, M. P. (2007). Sleep-dependent memory consolidation and reconsolidation. Sleep Med. 8, 331-343.

Stickgold, R., Whidbee, D., Schirmer, B. Patel, V., and Hobson, J. A. (2000). Visual discrimination task improvement: a multi-step process occurring during sleep. J. Cogn. Neurosci. 12, 246-254.

Tamaki, M., Matsuoka, T., Nittono, H., and Hori, T. (2008). Fast sleep spindle (13$15 \mathrm{~Hz}$ ) activity correlates with sleepdependent improvement in visuomotor performance. Sleep 31, 204-211.

Tandon, R., Shipley, J. E., Taylor, S. Greden, J. F., Eiser, A., DeQuardo, J., and Goodson, J. (1992). Electroencephalographic sleep abnormalities in schizophrenia. Relationship to positive/negative symptoms and prior neuroleptic treatment. Arch Gen. Psychiatry 49, 185-194.

Traub, A. C. (1972). Sleep stage deficits in chronic schizophrenia. Psychol. Rep. $31,815-820$.

Turek, F. W. (2005). Insomnia and depression: if it looks and walks like a duck. Sleep 28, 1362-1363.

Tyler, D. B. (1955). Psychological changes during experimental sleep deprivation. Dis. Nerv. Syst. 16, 293-299.
Van Dongen, H. P., Maislin, G., Mullington, J. M., and Dinges, D. F. (2003). The cumulative cost of additional wakefulness: dose-response effects on neurobehavioral functions and sleep physiology from chronic sleep restriction and total sleep deprivation. Sleep 26, 117-126.

Wagner,U., Gais, S., Haider, H., Verleger, R., and Born, J. (2004). Sleep inspires insight. Nature 427, 352-355.

Walker, M. P., Brakefield, T., Morgan, A., Hobson, J.A., and Stickgold, R. (2002). Practice with sleep makes perfect: sleep-dependent motor skill learning. Neuron 35, 205-211.

Walker, M. P., Stickgold, R., Alsop, D., Gaab, N., and Schlaug, G. (2005) Sleep-dependent motor memory plasticity in the human brain. Neuroscience 133, 911-917.

Wehr, T. A., Sack, D. A., and Rosenthal, N. E. (1987). Sleep reduction as a final common pathway in the genesis of mania. Am. J. Psychiatry 144, 201-204.

Wilson, M. A., and McNaughton, B. L. (1994). Reactivation of hippocampal ensemble memories during sleep. Science 265, 676-679.

Wright, J. B. (1993). Mania following sleep deprivation. Br. J. Psychiatry $163,679-680$

Yang, C., and Winkelman, J. W. (2006). Clinical significance of sleep EEG abnormalities in chronic schizophrenia. Schizophr. Res. 82, 251-260.

Conflict of Interest Statement: The authors declare that the research was conducted in the absence of any commercial or financial relationships that could be construed as a potential conflict of interest.

Received: 30 June 2009; paper pending published: 06 August 2009; accepted: 12 August 2009; published online: 01 September 2009.

Citation: Manoach DS and Stickgold $R$ (2009) Does abnormal sleep impair memory consolidation in schizophrenia? Front. Hum. Neurosci. 3:21. doi: 10.3389/neuro.09.021.2009

Copyright $\odot 2009$ Manoach and Stickgold. This is an open-access article subject to an exclusive license agreement between the authors and the Frontiers Research Foundation, which permits unrestricted use, distribution, and reproduction in any medium, provided the original authors and source are credited. 